\title{
Vascular Response Occurring at 3 Months After Everolimus-Eluting Cobalt-Chromium Stent Implantation in Patients With ST-Segment Elevation Myocardial Infarction vs. Stable Coronary Artery Disease
}

\author{
Masaru Ishida, MD; Daisuke Terashita, MD; Tomonori Itoh, MD; Hiromasa Otake, MD; \\ Yoshiro Tsukiyama, MD; Tatsuo Kikuchi, MD; Takatoshi Hayashi, MD; \\ Takahide Suzuki, MD; Yoshiaki Ito, MD; Takashi Morita, MD; Kiyoshi Hibi, MD; \\ Takahiro Sawada, MD; Takayuki Okamura, MD; Junya Shite, MD; \\ Fumiaki Takahashi, PhD; Toshiro Shinke, MD; Yoshihiro Morino, MD
}

\begin{abstract}
Background: Second-generation drug-eluting stents (DES) reduce the incidence of stent thrombosis, even in patients with ST-segment elevated myocardial infarction (STEMI). However, the early local vascular healing after DES implantation in STEMI lesions, which mainly concerns stent thrombosis, is still unclear.
\end{abstract}

\begin{abstract}
Methods and Results: We attempted to determine early local vascular healing 3 months after cobalt-chromium everolimus-eluting stent (CoCr-EES) implantation in STEMI lesions relative to stable coronary artery disease (CAD) lesions. This prospective, multicenter study analyzed 96 total lesions (STEMI=49, stable $C A D=51$ ) by frequency domain-optical coherence tomography (FD-OCT) performed post-procedure and at the 3-month follow-up. Although CoCr-EES implanted in STEMI were almost entirely covered at 3 months, they had a relatively high incidence of uncovered struts compared with stable CAD $(5.5 \%$ vs. $1.6 \%, P<0.001)$. Intrastent thrombus in the 2 groups was primarily resolved at the 3-month follow-up (STEMI: $91.7 \% \rightarrow 26.5 \%$, stable CAD: $74.5 \% \rightarrow 11.8 \%$ ). Regarding irregular protrusion, complete resolution was observed in stable CAD $(21.6 \% \rightarrow 0 \%)$, while a few stents remained in STEMI $(79.2 \% \rightarrow 8.2 \%)$. Although there were almost no changes for the serial change of average lumen area in STEMI, there were slight but significant decreases in stable CAD [STEMI $0.08(-0.44,0.55) \mathrm{mm}^{2}$, stable CAD $-0.35(-0.55,0.11) \mathrm{mm}^{2} ; \mathrm{P}=0.009$ ].
\end{abstract}

Conclusions: Although strut coverage after CoCr-EES implantation for STEMI lesions was slightly delayed, the healing process appeared to be acceptable in both STEMI and stable CAD.

Key Words: Coronary artery disease; Drug-eluting stent (DES); Optical coherence tomography (OCT); ST-segment elevation myocardial infarction

$\mathbf{S}$ tent thrombosis (ST) rarely occurs in patients undergoing 2nd-generation drug-eluting stent (DES) implantation for coronary artery disease (CAD), although it remains a critical complication. ${ }^{1}$ Patients with acute myocardial infarction (AMI) especially have a relatively high incidence of ST as compared with stable CAD patients. ${ }^{2}$ It has also been demonstrated that ST mainly occurs within 3 months after DES implantation in AMI patients and that delayed vessel healing at the thrombotic lesion is observed within 30 days after DES implantation. ${ }^{1,36}$ Thus, rapid progression of vascular healing during the early phase, together with thromboresistance, is the desired outcome of DES for AMI patients.

Cobalt-chromium everolimus-eluting stent (CoCr-EES),

Received March 26, 2020; revised manuscript received July 10, 2020; accepted July 15, 2020; J-STAGE Advance Publication released online October 2, 2020 Time for primary review: 45 days

Iwate Medical University, Iwate (M.I., T.I., F.T., Y.M.); Kobe University Graduate School of Medicine, Kobe (D.T., H.O., Y.T., T. Shinke); Edogawa Hospital, Tokyo (T.K.); Awaji Medical Center, Sumoto (T.H.); Hokkaido Welfare Federation of Agricultural Cooperative Engaru Kosei General Hospital, Hokkaido (T. Suzuki); Saiseikai Yokohama City Eastern Hospital, Yokohama (Y.I.); Osaka General Medical Center, Osaka (T.M.); Yokohama City University Medical Center, Yokohama (K.H.); Himeji Cardiovascular Center, Himeji (T. Sawada); Yamaguchi University Graduate School of Medicine, Ube (T.O.); Osaka Saiseikai Nakatsu Hospital, Osaka (J.S.); and Showa University School of Medicine, Tokyo (T. Shinke), Japan

The first two authors contributed equally to this work (M.I., D.T.).

Mailing address: Tomonori Itoh, MD, Iwate Medical University, 2-1-1 Idaidori, Yahaba-cho, Shiwa-gun, Iwate 028-3695, Japan. E-mail: tomoitoh@iwate-med.ac.jp

All rights are reserved to the Japanese Circulation Society. For permissions, please e-mail: cj@j-circ.or.jp

ISSN-1346-9843 
which is a representative of the 2nd-generation DES, has been shown to result in a lower incidence of early and late ST in patients with ST-segment elevation myocardial infarction (STEMI) as compared with bare metal stent (BMS) or a 1st-generation DES. ${ }^{5,7}$ However, it has yet to be determined whether there is early local vascular healing following CoCr-EES implantation in STEMI lesions. Based on previous clinical evidence, we hypothesized there would excellent strut coverage in the STEMI lesion at 3 months after CoCr-EES implantation as well as stable CAD lesions. In order to confirm this hypothesis, the MECHANISM (Multicenter Comparison of Early and Late Vascular Responses to Everolimus-eluting CobaltCHromium Stent and platelet AggregatioN studIeS for TreatMent of Acute Myocardial Infarction) study, which was a multicenter registry of serial frequency domain-optical coherence tomography (FD-OCT) imaging for STEMI or stable CAD lesions, was designed to assess early vascular healing following CoCr-EES implantation. In the current study, we attempted to determine the presence of early local vascular healing during the 3 months after CoCr-EES implantation in STEMI lesions as compared with stable CAD lesions.

\section{Methods}

The MECHANISM studies, which were of a prospective, multicenter registry of patients undergoing serial FD-OCT imaging with a quadruple study arm (MECHANISMAMI-2 W and -3M; ClinicalTrials.gov ID: NCT02014753, UMINID: UMIN000012616, MECHANISM-Elective$1 \mathrm{M}$ and -3M; ClinicalTrials.gov ID: NCT 02014818, UMINID: UMIN000014440), were performed in order to determine the presence of early or super-early vascular healing following CoCr-EES implantation. Of these study arms, the results of the MECHANISM-AMI-2 W study and MECHANISM-elective- $1 \mathrm{M}$ and $-3 \mathrm{M}$ studies have been reported in detail.8,9 In this study, we performed prespecified analysis for each of the 3-month follow-up arms and post-hoc comparison analysis between the MECHANISM-AMI-3M and MECHANISM-Elective$3 \mathrm{M}$ studies. All patients provided written informed consent prior to being admitted to the study. This study complies with the guidelines of the Declaration of Helsinki, and the ethical committee of each participating institution approved the study protocol.

\section{Subjects and Procedures}

Patients were recruited between April 2014 and June 2015 from 17 Japanese institutions. STEMI patients who met the diagnostic criterion for the Third Universal Definition of Myocardial Infarction were included in the MECHANISM-AMI study. ${ }^{10}$ Stable CAD patients, who were defined as having a positive stress test and no change in the frequency, duration, or intensity of symptoms within 4 weeks, were included in the MECHANISM-Elective study. Exclusion criteria were: cardiogenic shock, culprit lesion of the left main coronary artery, reference vessel diameter $<2.0 \mathrm{~mm}$ or $\geq 4.5 \mathrm{~mm}$, chronic kidney disease as indicated by serum creatinine $>2.0 \mathrm{mg} / \mathrm{dL}$, maintenance hemodialysis, comorbid cancer with expected survival $<2$ years, scheduled surgery within 3 months, history of adverse reactions to aspirin or clopidogrel, warfarin intake before STEMI onset, age $<20$ years, pregnancy, and STEMI at a prior stented segment.
Culprit lesions were treated with either 1 or $2 \mathrm{CoCr}-$ EESs (Xience Prime/Xpedition, Abbott Vascular, Santa Clara, CA, USA). Immediately after the procedure, FDOCT image acquisition was performed throughout the stented segment, with a margin segment $\geq 5 \mathrm{~mm}$ from the culprit lesion. For STEMI patients and stable patients without antiplatelet therapy, a loading dose of aspirin $(81-200 \mathrm{mg})$ and either clopidogrel $(300 \mathrm{mg})$ or prasugrel $(20 \mathrm{mg})$ (standard Japanese loading doses) were administered before the percutaneous coronary intervention (PCI). Dual antiplatelet therapy (DAPT) with aspirin and thienopyridine was continued at least until the end of the 3-month follow-up.

\section{FD-OCT Imaging and Analysis}

Coronary imaging was performed immediately after the PCI, and at 3 months ( $90 \pm 30$ days) during the staged PCI for residual stenotic lesions or diagnostic catheterization. Intracoronary injection of isosorbide dinitrate or nitroglycerin was administered prior to the FD-OCT imaging. FD-OCT images were acquired with an OCT system (ILUMIEN or ILUMIEN OPTIS) and a FD-OCT catheter (Dragonfly/Dragonfly JP/Dragonfly OPTIS; Abbott Vascular). All FD-OCT images of the target lesion site (coronary segment from $\geq 5 \mathrm{~mm}$ distal and $5 \mathrm{~mm}$ proximal to the target lesion) were recorded using an automatic pullback system. Although the use of a contrast agent as a flushing liquid was recommended, lactated Ringer's solution or low-molecular-weight dextran $\mathrm{L}$ were also considered to be acceptable. FD-OCT images were digitally stored and submitted for offline analyses.

FD-OCT image analysis was performed by 2 independent core laboratories (MECHANISM-AMI: Kobe Cardiovascular Core Laboratory, Kobe, Japan, MESHANISMElective: Iwate Core-analysis Laboratory, Morioka, Japan) using ILUMIEN proprietary software (Abbott Vascular). Each core laboratory confirmed all of the FD-OCT measurements that were reviewed by the other laboratories and used to build consensus for any questionable images.

Quantitative FD-OCT analyses were performed for every 1-mm interval in order to assess the lumen, stent, and intrastent tissue area (stent area-lumen area (LA)). Intrastent tissue thickness was measured from the lumen border to the center of the strut blooming. Struts with an intrastent tissue thickness $<0 \mu \mathrm{m}$ were defined as uncovered struts. A strut that was partially covered with tissue was classified as an uncovered strut. Because strut thickness determined by OCT imaging is considered to be the distance adding the actual strut thickness and polymer thickness to the OCT resolution limit $(81+7+20=108 \mathrm{~mm}),{ }^{11}$ a maximum distance $>108 \mu \mathrm{m}$ between the center reflection of the strut and the adjacent vessel surface was defined as a malapposed strut. The $\%$ uncovered and $\%$ malapposed struts were calculated as a percentage of the total number of uncovered or malapposed struts divided by the total number of struts. Between both core laboratories, the inter-laboratory $\kappa$ coefficient for uncovered struts was 0.80 . The intralaboratory $\kappa$ coefficients for uncovered struts were 0.97 (Iwate) and 0.92 (Kobe), respectively.

Qualitative FD-OCT analyses were performed for every single frame in order to assess intrastent thrombus, irregular protrusion and intrastent dissection, in accordance with previously published methods. ${ }^{12-15}$ The maximal area was measured for each mass or segment individually. Edge dissection was defined as disruption of the arterial lumen 


\begin{tabular}{|c|c|c|c|}
\hline & $\begin{array}{l}\text { STEMI } \\
(n=49)\end{array}$ & $\begin{array}{c}\text { Stable CAD } \\
(n=47)\end{array}$ & $P$ value \\
\hline Age (years) & $63(54,70)$ & $70(64,77)$ & $<0.001$ \\
\hline Male & $40(82)$ & $38(81)$ & 0.92 \\
\hline Hypertension & $32(65)$ & $40(85)$ & 0.03 \\
\hline Dyslipidemia & $32(65)$ & $39(83)$ & 0.049 \\
\hline Diabetes mellitus & $15(31)$ & $29(62)$ & $<0.005$ \\
\hline Smoking & $28(57)$ & $18(38)$ & 0.07 \\
\hline Prior $\mathrm{PCl}$ & $1(2)$ & $19(40)$ & $<0.001$ \\
\hline Prior MI & $1(2)$ & $14(30)$ & $<0.001$ \\
\hline \multicolumn{4}{|c|}{ Laboratory data on admission } \\
\hline Creatinine (mg/dL) & $0.76(0.66,0.88)$ & $0.84(0.69,1.08)$ & 0.04 \\
\hline LDL-C (mg/dL) & $137(112,166)$ & $85(74,121)$ & $<0.001$ \\
\hline $\mathrm{HDL}-\mathrm{C}(\mathrm{mg} / \mathrm{dL})$ & $44(40,54)$ & $49(39,57)$ & 0.58 \\
\hline Triglyceride (mg/dL) & $127(72,187)$ & $115(87,157)$ & 0.71 \\
\hline \multicolumn{4}{|l|}{ Drugs during follow-up } \\
\hline Statin & $45(94)$ & $36(77)$ & 0.02 \\
\hline Aspirin & $52(100)$ & $49(100)$ & - \\
\hline Thienopyridine & $48(100)$ & $46(98)$ & 0.31 \\
\hline
\end{tabular}

Values are $\mathrm{n}(\%)$ or median (1 st, 3rd quartiles). CAD, coronary artery disease; HDL-C, high-density lipoprotein cholesterol; LDL-C, low-density lipoprotein cholesterol; STEMI, ST-segment elevation myocardial infarction.

\begin{tabular}{|lccc|}
\hline Table 2. Target Vessel and Procedural Characteristics & & & \\
& $\begin{array}{c}\text { STEMI } \\
(\mathbf{n}=\mathbf{4 9})\end{array}$ & $\begin{array}{c}\text { Stable CAD } \\
\mathbf{( n = 4 7 )}\end{array}$ & P value \\
Target vessel characteristics & $21 / 8 / 20$ & $25 / 9 / 13$ & 0.40 \\
LAD/LCX/RCA & $11(23)$ & $14(31)$ & 0.37 \\
Bifurcation & & & 0.11 \\
Procedural characteristics & $3.0(2.75,3.5)$ & $3.0(2.5,3.25)$ & 0.94 \\
Stent diameter $(\mathrm{mm})$ & $28(18,33)$ & $27(18,33)$ & 0.58 \\
Total stent length $(\mathrm{mm})$ & $34(70)$ & $35(75)$ & 0.45 \\
Post dilation & $16(14,18)$ & $14(14,18)$ & \\
Maximum inflation pressure $(\mathrm{mmHg})$ &
\end{tabular}

Values are $\mathrm{n}(\%)$ or median (1 st, 3rd quartiles). CAD, coronary artery disease; $\mathrm{LAD}$, left anterior descending artery; LCX, left circumflex; RCA, right coronary artery; STEMI, ST-segment elevation myocardial infarction.

surface in the stent edge segment that was seen in at least 2 consecutive cross-section images. ${ }^{16}$

The primary endpoint of this study was the incidence of an uncovered strut at the 3-month follow-up. Secondary endpoints were the incidence of malapposed struts and intrastent findings including intrastent thrombus, irregular protrusion and intrastent dissection at the 3-month follow-up.

\section{Statistical Analysis}

Because most of the continuous values were not normally distributed, they are presented as medians with the 1st and 3rd quartile and then compared between the STEMI and stable CAD patients using Mann-Whitney U tests. Wilcoxon signed-rank tests were performed for comparisons between post-procedure and the 3-month follow-up. Categorical variables are presented as number or frequency, and compared using the chi-square test or Fisher's exact test, as appropriate. In terms of primary and secondary endpoints, we constructed a 2nd model using propensity score (PS) matching in order to adjust for clinical backgrounds. PS were estimated using a multivariate logistic regression model, which included age, sex, hypertension, dyslipidemia, diabetes mellitus and current smoking. Matching was performed with the use of 1:1 matching protocol without replacement (greedy-matching algorithm), with a caliper width equal to 0.2 of the standard deviation of the logit of the PS. P values $<0.05$ were considered statistically significant. All statistical analyses were performed using SPSS 24.0 (SPSS, Inc., Chicago, IL, USA).

\section{Results}

This prospective multicenter study enrolled 50 lesions in 50 STEMI patients and 55 lesions in 51 stable CAD patients. Due to poor-quality FD-OCT images, 1 STEMI patient and 2 stable CAD patients were excluded, and 2 stable CAD patients were excluded because they withdrew their consent. After these exclusions, there were 49 lesions in 49 STEMI patients and 51 lesions in 47 CAD patients that were analyzed by comparing serial FD-OCT images obtained between the index procedure and the 3-month 


\begin{tabular}{|c|c|c|c|}
\hline & $\begin{array}{l}\text { STEMI } \\
(\mathrm{n}=49)\end{array}$ & $\begin{array}{c}\text { Stable CAD } \\
(n=47)\end{array}$ & $P$ value \\
\hline Stent length & $22.2(15.7,29.2)$ & $22.0(16.8,30.9)$ & 0.84 \\
\hline Total stent struts & $189(156,278)$ & $217(165,294)$ & 0.55 \\
\hline Average stent area $\left(\mathrm{mm}^{2}\right)$ & $6.95(5.79,8.49)$ & $5.89(5.26,7.05)$ & 0.03 \\
\hline Minimum stent area $\left(\mathrm{mm}^{2}\right)$ & $5.35(4.36,6.50)$ & $4.73(3.92,6.35)$ & 0.24 \\
\hline Average LA $\left(\mathrm{mm}^{2}\right)$ & $6.61(5.86,8.42)$ & $6.02(5.31,6.99)$ & 0.07 \\
\hline Minimum LA (mm²) & $5.09(4.09,6.10)$ & $4.70(3.84,6.04)$ & 0.46 \\
\hline$\%$ Uncovered struts & $62.1(48.2,76.9)$ & $82.7(69.9,88.5)$ & $<0.001$ \\
\hline$\%$ Malapposed struts & $3.8(1.3,8.2)$ & $5.2(2.9,11.6)$ & 0.29 \\
\hline Incidence of intrastent thrombus & 91.7 & 74.5 & 0.24 \\
\hline No. of intrastent thrombi & $3(1,4)$ & $1(0,2)$ & $<0.001$ \\
\hline Maximum area of intrastent thrombus $\left(\mathrm{mm}^{2}\right)$ & $0.66(0.26,1.18)$ & $0.22(0,0.46)$ & $<0.001$ \\
\hline Incidence of irregular protrusions & 79.2 & 21.3 & $<0.001$ \\
\hline No. of irregular protrusions & $1(0,2)$ & $0(0,0)$ & 0.047 \\
\hline Maximum area of irregular protrusion $\left(\mathrm{mm}^{2}\right)$ & $0.38(0.04,0.78)$ & $0(0,0)$ & $<0.001$ \\
\hline Incidence of any edge dissection & 25.0 & 21.3 & 0.43 \\
\hline
\end{tabular}

Values are \% or median (1 st, 3rd quartiles). CAD, coronary artery disease; LA, lumen area; PCI, percutaneous coronary intervention; STEMI, ST-segment elevation myocardial infarction.

\begin{tabular}{|lccc|}
\hline \multicolumn{1}{|l|}{ Table 4. Comparison of OCT Measurements at 3-Month Follow-up } & & \\
& $\begin{array}{c}\text { STEMI } \\
(\mathbf{n = 4 9 )}\end{array}$ & $\begin{array}{c}\text { Stable CAD } \\
(\mathbf{n}=\mathbf{4 7})\end{array}$ & P value \\
Stent length & $21.8(16.8,28.8)$ & $22.4(15.7,29.2)$ & 0.96 \\
Total stent struts & $219(164,295)$ & $228(158,305)$ & 0.78 \\
Average stent area $\left(\mathrm{mm}^{2}\right)$ & $7.30(5.91,8.76)$ & $5.89(4.93,7.13)$ & 0.01 \\
Minimum stent area $\left(\mathrm{mm}^{2}\right)$ & $5.35(4.32,6.90)$ & $4.45(3.97,6.12)$ & 0.054 \\
Average LA $\left(\mathrm{mm}^{2}\right)$ & $6.83(5.76,8.48)$ & $5.67(4.72,7.01)$ & 0.02 \\
Minimum LA $\left(\mathrm{mm}^{2}\right)$ & $5.13(3.84,6.23)$ & $4.38(3.66,5.95)$ & 0.08 \\
Neointimal thickness $(\mu \mathrm{m})$ & $55(43,73)$ & $40(30,58)$ & $<0.001$ \\
\% Uncovered struts & $5.5(2.2,9.7)$ & $1.6(0,2.8)$ & $<0.001$ \\
\% Malapposed struts & $0(0,1.5)$ & $0(0,2.0)$ & 0.30 \\
Incidence of intrastent thrombus & 26.5 & 12.8 & 0.08 \\
No. of intrastent thrombi & $0(0,1)$ & $0(0,0)$ & 0.06 \\
Maximum area of intrastent thrombus $\left(\mathrm{mm}^{2}\right)$ & $0(0,0.16)$ & $0(0,0)$ & 0.056 \\
Incidence of irregular protrusions & 8.2 & 0 & 0.06 \\
No. of irregular protrusions & $0(0,0)$ & $0(0,0)$ & 0.047 \\
Maximum area of irregular protrusion $\left(\mathrm{mm}^{2}\right)$ & $0(0,0)$ & $0(0,0)$ & 0.047 \\
Incidence of any edge dissection & 4.1 & 10.6 & 0.20 \\
\hline
\end{tabular}

Values are \% or median (1 st, 3rd quartiles). CAD, coronary artery disease; LA, lumen area; OCT, optical coherence tomography; STEMI, ST-segment elevation myocardial infarction.

follow-up. During the 3 months, neither cardiac death nor ST occurred in either group.

\section{Patient and Procedural Characteristics}

Table 1 presents the patients' characteristics. Compared with the stable CAD patients, STEMI patients were younger and had less hypertension, dyslipidemia and diabetes mellitus. In addition, the mean low-density lipoprotein cholesterol level in STEMI patients was significantly higher than that observed in the stable CAD patients. There were no differences between the 2 groups for the target vessel and procedural characteristics, including stent diameter and length (Table 2). In the PS-matched model, either patients or procedural characteristics were comparable between
STEMI and stable CAD (Supplementary Table 1).

\section{Post-Procedure FD-OCT Images}

Table 3 presents the comparison of the post-procedure FD-OCT findings between the 2 groups. Although the minimum stent areas were similar, the average stent area in STEMI was larger than that observed in stable CAD. Furthermore, the STEMI lesions had more frequent and larger intra-stent thrombus as compared with stable CAD lesions. In addition, the STEMI lesions were more frequent and had larger irregular protrusions as well as intrastent thrombus. The incidence of uncovered struts in STEMI was lower than that observed in stable CAD (unadjusted model: $62.1 \%$ vs. $82.7 \% \mathrm{P}<0.001$, PS-matching model: 
A: Uncovered struts (Unadjusted model)

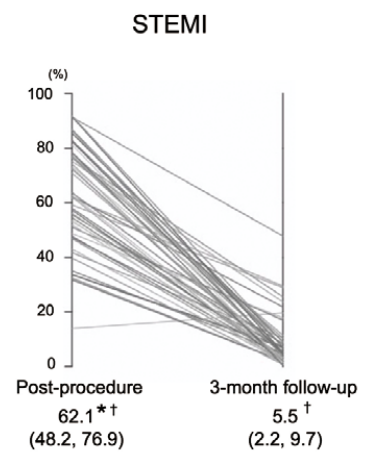

C: Uncovered struts (PS matched model)

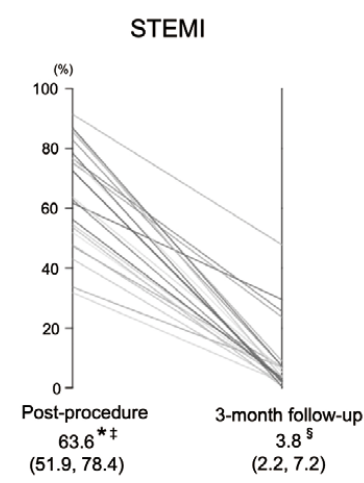

Stable CAD

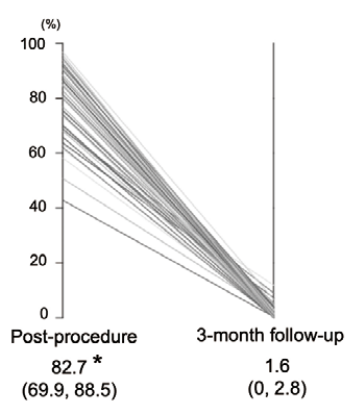

B: Malapposed struts (Unadjusted model)

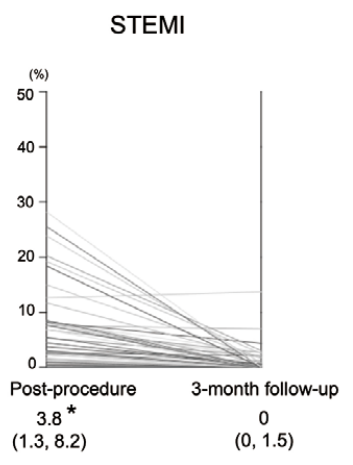

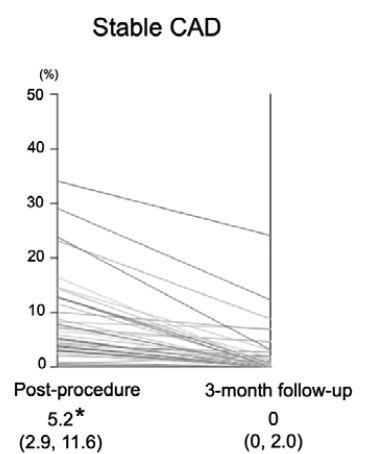

\section{D: Malapposed struts (PS matched model)}
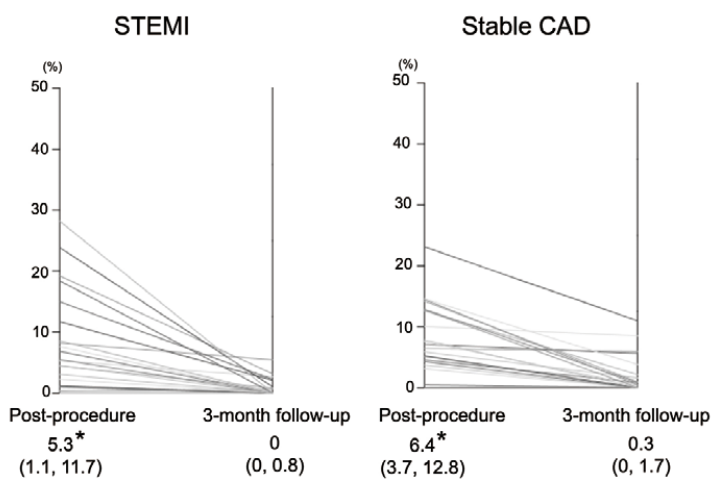

Figure 1. (A-D) Serial changes in the incidence of uncovered and malapposed struts in STEMI lesions vs. Stable CAD lesions. ${ }^{*}$ Post vs. 3 months: $P<0.001$; †STEMI vs. Stable CAD: $P<0.001$; $\neq S T E M I$ vs. stable $C A D: P=0.01$; $\$ S T E M I$ vs. stable $C A D: P=0.001$. CAD, coronary artery disease; STEMI, ST-segment elevation myocardial infarction.

$63.6 \%$ vs. $81.4 \% \mathrm{P}=0.01$ ). On the other hand, there was no difference in the incidence of malapposed struts between the 2 groups.

\section{Follow-up FD-OCT Images}

Table 4 shows the comparison of the FD-OCT findings between the 2 groups at 3-month follow-up. Compared with the stable CAD lesions, the STEMI lesions maintained a relatively large average LA. Although CoCr-EES implanted in STEMI lesions were most likely covered at 3 months, there was a relatively high incidence of uncovered struts compared with that seen for the stable CAD lesions $(5.5 \%$ vs. $1.6 \%, \mathrm{P}<0.001)$ and this result was confirmed in the PS-matching model $(3.8 \%$ vs. $1.7 \%, \mathrm{P}=0.001)$ (Supplementary Table 2). In contrast, there was resolution of almost all of the malapposed struts in the 2 groups. Intrastent thrombus and irregular protrusion were mostly resolved at 3 months. STEMI lesions had, however, relatively less intrastent dissection ( $10.2 \%$ vs. $25.2 \%, \mathrm{P}=0.04)$, and tended to have more intrastent thrombus $(26.5 \%$ vs. $12.8 \%, \mathrm{P}=0.08)$ and irregular protrusion $(8.2 \%$ vs. $0 \%$, $\mathrm{P}=0.06)$ as compared with stable CAD lesions. In STEMI lesions, the median neointimal thickness was thicker than that observed in stable CAD $(55 \mu \mathrm{m}$ vs. $40 \mu \mathrm{m}$, $\mathrm{P}<0.001)$. However, this statistical difference disappeared after the PS matching $(54 \mu \mathrm{m}$ vs. $37 \mu \mathrm{m}, \mathrm{P}=0.07)$ (Supplementary Table 2).

\section{Comparison of Post-Procedure and Follow-up FD-OCT Images}

Compared with the post-procedure FD-OCT findings, there was a significant decrease in the incidence of uncovered struts at the 3-month follow-up in both STEMI lesions $(\mathrm{P}<0.001)$ and stable CAD lesions $(\mathrm{P}<0.001)$ (Figure 1). In addition, during the 3 months following CoCr-EES implantation, there was resolution of almost all of the malapposed struts in both STEMI $(\mathrm{P}<0.001)$ and stable CAD lesions $(\mathrm{P}<0.001)$. Similarly, the average maximum area of intrastent thrombus (STEMI: $\mathrm{P}<0.001$, stable CAD: $\mathrm{P}<0.001$ ) and irregular protrusion (STEMI: $\mathrm{P}<0.001$, stable CAD: $P=0.003)$ in the 2 groups were reduced to almost zero at the 3-month follow-up (Figure 2). Residual irregular protrusion at 3 months after stent implantation was especially not observed in stable CAD lesions, whereas 4 STEMI lesions did exhibit residual irregular protrusions at the 3-month follow-up. Most cases of stent edge dissection were resolved at 3 months following CoCr-EES implantation in both types of lesion: STEMI (post $25.0 \%, 3$ months $6.1 \%$ ) and stable CAD (post $19.6 \%$ vs. 3 months $9.8 \%$ ).

Figure 3 presents the serial changes in average and 
A: Intra-stent thrombus

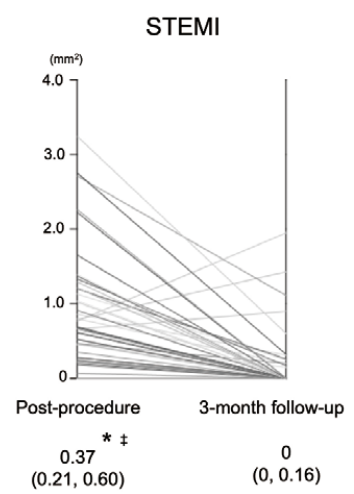

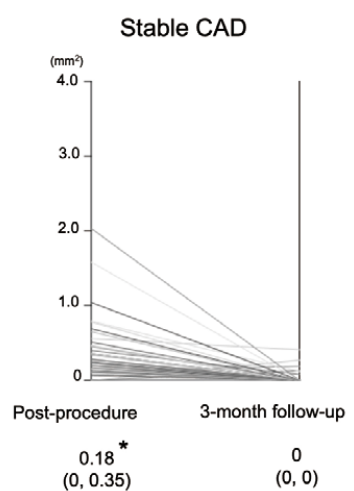

B: Irregular protrusion

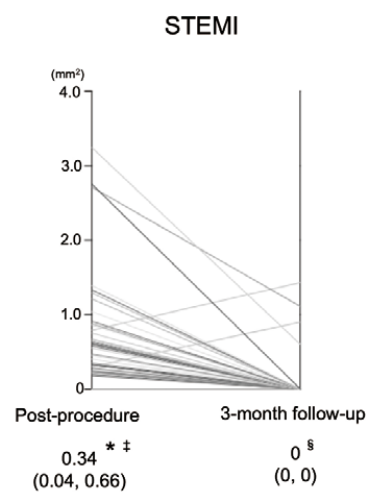

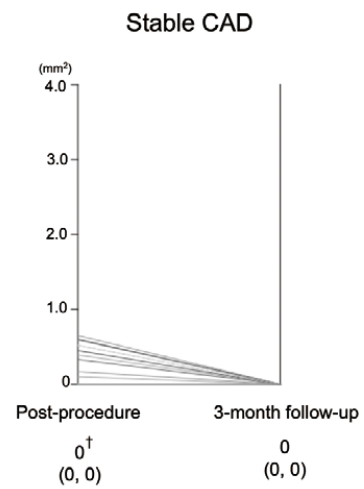

Figure 2. (A,B) Serial changes in the maximum area for each of the intrastent findings. *Post vs. 3 months: P<0.001; ${ }^{+} P \circ$ st vs. 3 months: $P<0.005$; $\neq S T E M I$ vs. Stable CAD: $P<0.001$; §STEMI vs. Stable CAD: $P<0.05$. CAD, coronary artery disease; STEMI, ST-segment elevation myocardial infarction.

A: Serial change of average LA

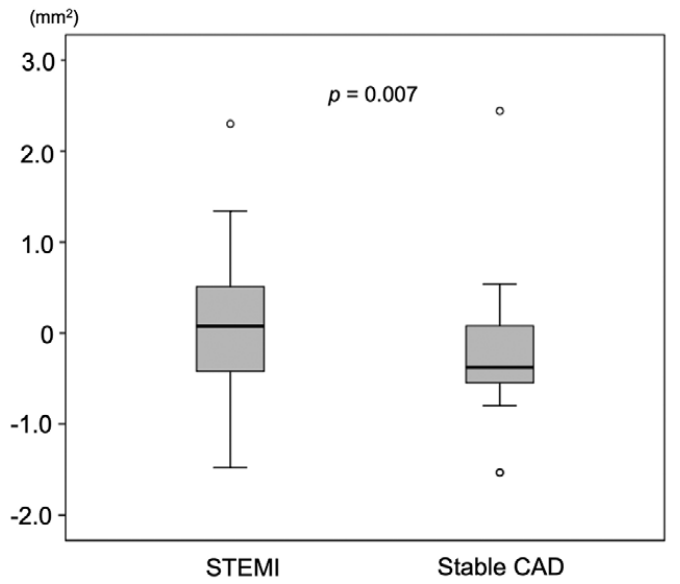

\section{B: Serial change of minimum LA}

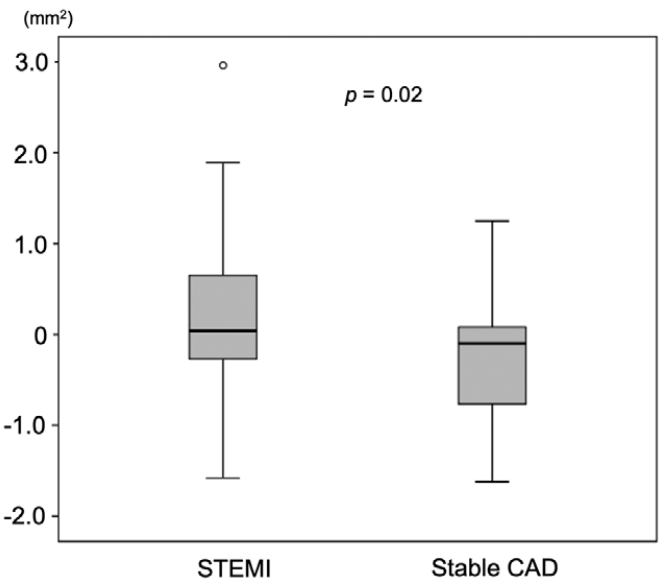

Figure 3. Serial changes in average and minimum lumen area (LA). CAD, coronary artery disease; STEMI, ST-segment elevation myocardial infarction.

minimum LA. There were almost no changes observed for either in the STEMI lesions, whereas there was a slight but relatively significant decrease of both in the stable CAD lesions relative to STEMI.

\section{Discussion}

In the present study, we assessed local vascular healing 3 months after CoCr-EES implantation in STEMI lesions as compared with stable CAD lesions. The main findings were as follows. (1) Although the incidence of uncovered struts at 3 months after CoCr-EES implantation in STEMI lesions was statistically higher than in stable CAD lesions, there was almost complete resolution of uncovered and malapposed struts at 3 months after CoCr-EES implantation in both STEMI and stable CAD lesions. (2) There was resolution of most cases of intrastent thrombus and irregular protrusion in the 2 groups at 3 months. (3) At the 3-month follow-up, STEMI lesions had a thicker neointima than stable CAD lesions, although this statistical difference disappeared after PS matching. (4) Between post-procedure and the 3-month follow-up, there was no difference in the FD-OCT findings for the average and minimum LA in the STEMI lesions, but both areas were significantly decreased in the stable CAD lesions.

Although there was a difference in the incidence of uncovered struts between STEMI and stable CAD (STEMI: $5.5 \%$ vs. stable CAD: $1.6 \%$ at 3 months, $\mathrm{P}<0.001$ ), the present study showed that the lesions at 3 months after CoCr-EES implantation exhibited greater strut coverage compared with previously reported data of sirolimus-eluting stent (acute coronary syndrome (ACS): 18\% vs. stable 
CAD: $13 \%) .{ }^{17}$ In addition, a recent study, in which one-third of the subjects underwent CoCr-EES implantation for ACS, showed resolution of most of the uncovered struts at 3-month follow-up (0.4-6.9\%). ${ }^{18,19}$ Because a previous pathological study has shown that rate of $>30 \%$ of uncovered struts is one of the best morphologic predictor of late ST, ${ }^{20}$ our present study results strongly support the safety of CoCr-EES for use in STEMI lesions.

According to experimental studies, the durable fluoropolymer that is the coating surface of the CoCr-EES exhibits acute thromboresistance relative to BMS or to contemporary DES with biodegradable coatings. ${ }^{21,22}$ Indeed, the present study demonstrated almost complete resolution of intrastent thrombus, even in STEMI lesions, at 3 months after CoCr-EES implantation. Based on previous evidence that one-third of lesions with 1st-generation DES have residual intrastent thrombus at $8-9$ months, ${ }^{23,24}$ the present study suggested that CoCr-EES has greater thromboresistance than 1st-generation DES.

To the best of our knowledge, the present study is the first report of temporal change in irregular protrusions, with $8.2 \%$ of STEMI lesions unresolved at 3 months after CoCr-EES implantation despite complete resolution in stable CAD lesions. Considering that lipid-rich and thrombotic lesions are related to the incidence of irregular protrusions, ${ }^{25}$ it is reasonable that STEMI lesions have more irregular protrusions than stable CAD lesions. Thus, the difference in underlying plaque morphology might be related to the incidence of irregular protrusions. Moreover, a previous study using near-infrared spectroscopy demonstrated a reduction in the size of lipid-rich plaque even at 7 weeks after intensive statin therapy. ${ }^{26}$ Because there is a high possibility that the main component of these irregular protrusions is lipid-rich plaque, resolution of irregular protrusions may be related to the size of the reduction in protruding lipid-rich plaque. Irregular protrusion assessed by post-procedure FD-OCT imaging is a current topic in interventional cardiology, ${ }^{14}$ but its effect on clinical outcome following stent implantation is controversial..$^{15,27}$ Therefore, it will be necessary to further investigate the clinical impact of residual irregular protrusion that is detected during follow-up FD-OCT.

Unlike the MECHANISM-AMI-2 W study, ${ }^{8}$ our data showed that there was no difference in the average and minimum LA after CoCr-EES implantation in STEMI lesions at the 3-month follow-up. Because of being thrombotic lesions, one-third of the stent struts in STEMI lesions were covered with thrombus or showed irregular protrusion. In addition, neointimal proliferation after CoCr-EES implantation linearly increases during the first 3 months. ${ }^{19}$ Thus, our results suggested that the area decreased by neointimal proliferation might become equal to the area increased by thrombus resolution at around 3 months following CoCr-EES implantation.

A previous OCT study reported that the neointimal volume at 6 months correlated with the volume of the post-procedural intrastent structures after biolimus-eluting stent implantation for STEMI. ${ }^{28}$ Similarly, the present study demonstrated a relatively thick neointima at 3 months in the STEMI lesions vs. the stable CAD lesions. Although the difference between STEMI and stable CAD was ambiguous in the matched cohort, this was probably due to the low statistical power. However, the early neointima formation that takes place after implantation of current- generation DES in STEMI lesions may be more advanced than that observed in stable CAD lesions.

For patients at high bleeding risk, current guidelines from the American College of Cardiology/American Heart Association and the European Society of Cardiology recommend that DAPT be discontinued at 3 months after stent implantation for stable CAD, but a 6-month term of DAPT is recommended for STEMI. ${ }^{29}$ For STEMI patients with a high bleeding risk who undergo optimal medical therapy to prevent new atherosclerotic events, the feasible early vascular response of CoCr-EES shown in the present study provides supportive evidence for a 3-month term of DAPT for STEMI also.

\section{Study Limitations}

First, as this study compared different registries, it was not possible to perform formal sample size calculations. Second, only small sample sizes were used to assess the clinical outcomes, including ST. A subsequent study with larger cohorts and longer-term follow-up will need to be undertaken in order to confirm the association between FD-OCT findings and the clinical outcomes. Third, as the majority of the patients included in this study had multiple coronary vessel disease, there could have been a potential selection bias in either the STEMI or stable CAD groups.

\section{Conclusions}

Although there was a slight delay in strut coverage after CoCr-EES implantation for STEMI lesions, the healing process appeared to be acceptable in both the STEMI and stable CAD groups. This result suggests that CoCr-EES implantation for STEMI could be feasible and has a low incidence of ST relative to BMS or 1st-generation DES.

\section{Acknowledgments}

The authors are deeply grateful to the core-analysis laboratory members: Kanako Omiya and Yumiko Okuyama, Kayoko Fujiwara (secretary) and Tatsuya Shinke (image technician).

\section{Funding}

This study was supported by Abbott Vascular Inc. (Santa Clara, CA, USA) and Daiichi-Sankyo Co. Ltd. (Tokyo, Japan).

\section{Conflicts of Interest}

M.I. receives lecture honoraria from Abbott Vascular, Amgen Astellas BioPharma, Boston Scientific and Daiichi-Sankyo. Y.I. receives lecture honoraria from Daiichi-Sankyo, and Abbott Vascular. H.O. is consultant to and receiving grant/research support from Abbott Vascular, Daiichi-Sankyo and Terumo. T. Shinke, Y.M., and all other authors do not have any conflicts of interest or financial disclosures.

\section{Data Availability}

The deidentified participant data will not be shared.

\section{Clinical Trial Registration}

NCT 02014818, UMINID: UMIN000014440.

\section{IRB Information}

Iwate Medical University, IRB Committee reference no. H25-119 (Mechanism-Elective study), H26-9 (Mechanism-AMI study).

\section{Disclosures}

Y.M. is a member of Circulation Journal' Editorial Team. 


\section{References}

1. Claessen BE, Henriques JP, Jaffer FA, Mehran R, Piek JJ, Dangas GD. Stent thrombosis: A clinical perspective. JACC Cardiovasc Interv 2014; 7: 1081 - 1092.

2. de la Torre-Hernandez JM, Alfonso F, Hernandez F, Elizaga J, Sanmartin M, Pinar E, et al. Drug-eluting stent thrombosis: Results from the multicenter Spanish registry ESTROFA (Estudio ESpanol sobre TROmbosis de stents FArmacoactivos). J Am Coll Cardiol 2008; 51: 986-990.

3. Nakazawa G, Finn AV, Joner M, Ladich E, Kutys R, Mont EK, et al. Delayed arterial healing and increased late stent thrombosis at culprit sites after drug-eluting stent placement for acute myocardial infarction patients: An autopsy study. Circulation 2008; 118: $1138-1145$.

4. Stone GW, Lansky AJ, Pocock SJ, Gersh BJ, Dangas G, Wong $\mathrm{SC}$, et al. Paclitaxel-eluting stents versus bare-metal stents in acute myocardial infarction. N Engl J Med 2009; 360: 1946-1959.

5. Sabate M, Cequier A, Iniguez A, Serra A, Hernandez-Antolin R, Mainar V, et al. Everolimus-eluting stent versus bare-metal stent in ST-segment elevation myocardial infarction (EXAMINATION): 1 year results of a randomised controlled trial. Lancet 2012; 380: $1482-1490$.

6. Aoki J, Kozuma K, Awata M, Nanasato M, Shiode N, Tanabe $\mathrm{K}$, et al. Five-year clinical outcomes of everolimus-eluting stents from the post marketing study of CoCr-EES (XIENCE V/ PROMUS) in Japan. Cardiovasc Interv Ther 2019; 34: 40-46.

7. Palmerini T, Biondi-Zoccai G, Della Riva D, Mariani A, Sabate $\mathrm{M}$, Valgimigli $\mathrm{M}$, et al. Clinical outcomes with drug-eluting and bare metal stents in patients with ST-segment elevation myocardial infarction: Evidence from a comprehensive network meta-analysis. J Am Coll Cardiol 2013; 62: 496-504.

8. Morino Y, Terashita D, Otake H, Kikuchi T, Fusazaki T, Kuriyama N, et al. Early vascular responses to everolimus-eluting cobalt-chromium stent in the culprit lesions of st-elevation myocardial infarction: Results from a multicenter prospective optical coherence tomography study (MECHANISM-AMI 2-week follow-up study). Cardiovasc Interv Ther 2018; 34: 14-24.

9. Shinke T, Itoh T, Ishida M, Otake H, Terashita D, Fusazaki T, et al. Early and mid-term vascular responses to optical coherence tomography-guided everolimus-eluting stent implantation in stable coronary artery disease. Can J Cardiol 2019; 35: 1513-1522.

10. Thygesen K, Alpert JS, Jaffe AS, Simoons ML, Chaitman BR, White HD, et al. Third universal definition of myocardial infarction. Circulation 2012; 126: 2020-2035.

11. Inoue T, Shite J, Yoon J, Shinke T, Otake H, Sawada T, et al Optical coherence evaluation of everolimus-eluting stents 8 months after implantation. Heart 2011; 97: 1379-1384.

12. Jang IK, Tearney GJ, MacNeill B, Takano M, Moselewski F, Iftima N, et al. In vivo characterization of coronary atherosclerotic plaque by use of optical coherence tomography. Circulation 2005; 111: $1551-1555$.

13. Gonzalo N, Serruys PW, Okamura T, Shen ZJ, Onuma Y, GarciaGarcia HM, et al. Optical coherence tomography assessment of the acute effects of stent implantation on the vessel wall: A systematic quantitative approach. Heart 2009; 95: 1913-1919.

14. De Cock D, Bennett J, Ughi GJ, Dubois C, Sinnaeve P, Dhooge J, et al. Healing course of acute vessel wall injury after drug-eluting stent implantation assessed by optical coherence tomography. Eur Heart J Cardiovasc Imaging 2014; 15: 800-809.

15. Soeda T, Uemura S, Park SJ, Jang Y, Lee S, Cho JM, et al. Incidence and clinical significance of poststent optical coherence tomography findings [Clinical Perspective]. Circulation 2015; 132: $1020-1029$.

16. Chamié D, Bezerra HG, Attizzani GF, Yamamoto H, Kanaya $\mathrm{T}$, Stefano GT, et al. Incidence, predictors, morphological characteristics, and clinical outcomes of stent edge dissections detected by optical coherence tomography. JACC: Cardiovascular Interventions 2013; 6: 800-813.

17. Takano M, Inami S, Jang IK, Yamamoto M, Murakami D, Seimiya K, et al. Evaluation by optical coherence tomography of neointimal coverage of sirolimus-eluting stent three months after implantation. Am J Cardiol 2007; 99: 1033-1038.

18. Kim JS, Kim JH, Shin DH, Kim BK, Ko YG, Choi D, et al. Serial randomized comparison of strut coverage of everolimusand first-generation sirolimus-eluting stents. Can J Cardiol 2015; 31: $723-730$.

19. Yano H, Horinaka S, Ishikawa M, Ishimitsu T. Early vascular responses after everolimus-eluting stent implantation assessed by serial observations of intracoronary optical coherence tomography. Heart Vessels 2017; 32: 804-812.

20. Finn AV, Joner M, Nakazawa G, Kolodgie F, Newell J, John $\mathrm{MC}$, et al. Pathological correlates of late drug-eluting stent thrombosis: Strut coverage as a marker of endothelialization. Circulation 2007; 115: 2435-2441.

21. Kolandaivelu K, Swaminathan R, Gibson WJ, Kolachalama VB, Nguyen-Ehrenreich KL, Giddings VL, et al. Stent thrombogenicity early in high-risk interventional settings is driven by stent design and deployment and protected by polymer-drug coatings. Circulation 2011; 123: 1400-1409.

22. Otsuka F, Cheng Q, Yahagi K, Acampado E, Sheehy A, Yazdani SK, et al. Acute thrombogenicity of a durable polymer everolimuseluting stent relative to contemporary drug-eluting stents with biodegradable polymer coatings assessed ex vivo in a swine shunt model. JACC Cardiovasc Interv 2015; 8: $1248-1260$.

23. Sawada T, Shinke T, Shite J, Honjo T, Haraguchi Y, Nishio R, et al. Impact of cytochrome P450 2C19*2 polymorphism on intra-stent thrombus after drug-eluting stent implantation in Japanese patients receiving clopidogrel. Circ J 2011; 75: 99-105.

24. Choi HH, Kim JS, Yoon DH, Hong KS, Kim TH, Kim BK, et al. Favorable neointimal coverage in everolimus-eluting stent at 9 months after stent implantation: Comparison with sirolimuseluting stent using optical coherence tomography. Int J Cardiovasc Imaging 2012; 28: $491-497$.

25. Bryniarski KL, Tahk SJ, Choi SY, Soeda T, Higuma T, Yamamoto $\mathrm{E}$, et al. Clinical, angiographic, IVUS, and OCT predictors for irregular protrusion after coronary stenting. EuroIntervention 2017; 12: e2204-e2211.

26. Dohi T, Maehara A, Moreno PR, Baber U, Kovacic JC, Limaye $\mathrm{AM}$, et al. The relationship among extent of lipid-rich plaque, lesion characteristics, and plaque progression/regression in patients with coronary artery disease: A serial near-infrared spectroscopy and intravascular ultrasound study. Eur Heart $J$ Cardiovasc Imaging 2015; 16: 81-87.

27. Maehara A, Matsumura M, Ali ZA, Mintz GS, Stone GW. IVUS-guided versus OCT-guided coronary stent implantation: A critical appraisal. JACC Cardiovasc Imaging 2017; 10: 14871503.

28. Garcia-Garcia HM, Muramatsu T, Nakatani S, Lee IS, Holm NR, Thuesen L, et al. Serial optical frequency domain imaging in STEMI patients: The follow-up report of TROFI study. Eur Heart J Cardiovasc Imaging 2014; 15: 987-995.

29. Capodanno D, Alfonso F, Levine GN, Valgimigli M, Angiolillo DJ. ACC/AHA versus ESC guidelines on dual antiplatelet therapy: JACC guideline comparison. J Am Coll Cardiol 2018; 72: 29152931.

\section{Supplementary Files}

Please find supplementary file(s); http://dx.doi.org/10.1253/circj.CJ-20-0264 